\title{
A New Generation Plasma FIB Column with Higher Probe Current and Improved Imaging Resolution
}

Tomáš Hrnčír ${ }^{1}$, Dušan Nešpor ${ }^{1}$, Zsolt Radi ${ }^{1}$, Jaroslav Jiruše ${ }^{1}$, Lukáš Hladík ${ }^{2}$, Olivier Salord ${ }^{3}$ and Anne Delobbe $^{3}$

${ }^{1}$ TESCAN Brno s.r.o, Brno, Jihomoravsky kraj, Czech Republic, ${ }^{2}$ TESCAN ORSAY HOLDING, a.s., Brno, Jihomoravsky kraj, Czech Republic, ${ }^{3}$ Orsay Physics, Fuveau, Provence-Alpes-Cote d'Azur, France

During past several years, plasma FIB has been established as a routine technique in semiconductor failure analysis and materials science. It offers a wide range of FIB applications like creating big and artefactfree cross sections [1-3], TEM sample preparation [4], 3D tomography [5, 6], integrated circuit delayering [7] and sample preparation for X-ray tomography [8]. However, there are still some limitations of the technique. When compared to Ga FIB, plasma FIB offers significantly faster milling, but its spot size is bigger [1]. This causes worsening of the cross-section quality. Moreover, there are still some applications which require higher beam currents than the maximum ones available now. There is also significantly increased interest in the low beam energy applications. These include e.g. TEM sample final polishing and surface preparation for EBSD. Integrated circuit delayering seems to be another very important low energy application. Due to the shrinkage of semiconductor devices, gentle FIB sputtering needs to replace too destructive mechanical polishing methods. It is possible to access individual layers and to create the surface suitable for nanoprobing, with minimized beam induced damage [7].

The new generation i-FIB+ column addresses above mentioned challenges. The column and its control electronics are redesigned completely. The maximum beam current is increased from $2 \mu \mathrm{A}$ to $3 \mu \mathrm{A}$ at the beam energy $30 \mathrm{keV}$, which brings $50 \%$ higher sputtering rate and reduces time for rough milling operations at the high current. The maximum field of view is increased to $1 \mathrm{~mm}$ at $30 \mathrm{keV}$, which together with the higher milling rate allows fabrication of bigger structures during individual milling operations. Higher FIB field of view, which is now closer to SEM field of view, also brings easier sample navigation. The imaging resolution at beam energy $30 \mathrm{keV}$ is improved from $25 \mathrm{~nm}$ to $12 \mathrm{~nm}$ (statistical method on many profiles), which makes Xe plasma FIB performance much closer to Ga FIB one. The low energy performance is also significantly improved by utilizing the new generation high voltage supply and optimization of the objective lens control. The spot shape is generally improved by redesigning the plasma source, by tuning the column operation conditions and by increasing the purity of Xe gas, precise microleak injection valve and precise pressure gauge installed on FIB gun. The beam limiting apertures are moved by piezo mechanism which is fast and perfectly repeatable. There are 30 apertures, to get longer lifetime of the aperture strip. It is very easy to exchange the aperture strip or individual apertures, when necessary. Generally, the serviceability of the column is easier now due to the detailed self-testing and error reporting.

The software control of the column brings several improvements too. FIB spot optimizer wizard helps to tune FIB spot shape and to obtain the best quality of the cross section at given input conditions (beam energy and current). 3D collision model allows to perform large movements of the sample stage and other movable parts inside the chamber, mitigating the risk of damaging the expensive equipment by potentially dangerous movements. Starting FIB and recalling the previously saved milling or imaging conditions requires just one button in the software now. The user is allowed to redesign the software interface and optimize the control for a given application task. 


\section{References}

[1] T. Hrnčír et al.; Novel plasma FIB/SEM for high speed failure analysis and real time imaging of large volume removal; ISTFA: Conference Proceedings from the 38th International Symposium for Testing and Failure Analysis (2012), 26.

[2] F. Altmann et al.; Cross Section Analysis of Cu Filled TSVs Based On High Throughput Plasma-FIB Milling; ISTFA: Conference Proceedings from the 38th International Symposium for Testing and Failure Analysis (2012), 39.

[3] T. Hrnčíř, M. Šikula and J. V. Oboňa; How to achieve artefact-free FIB milling on polyimide packages; ISTFA: Conference Proceedings from the 42nd International Symposium for Testing and Failure Analysis (2016), 642.

[4] T. Hrnčír et al.; How Xe and Ga FIB differ in inducing lateral damage on TEM samples; ISTFA: Conference Proceedings from the 41st International Symposium for Testing and Failure Analysis (2015), 60 .

[5] T. Hrnčír and L. Hladík; Fast and Precise 3D Tomography of TSV By Using Xe Plasma FIB; ISTFA: Conference Proceedings from the 39th International Symposium for Testing and Failure Analysis (2013), 27.

[6] T. L.Burnett et al.; Large volume serial section tomography by Xe Plasma FIB dual beam microscopy; Ultramicroscopy 161 (2016), 119.

[7] V. Viswanathan et al,; Precision Xe Plasma FIB Delayering for Physical Failure Analysis of sub-20 nm Microprocessor Devices; ISTFA: Conference Proceedings from the 43rd International Symposium for Testing and Failure Analysis (2017), 593.

[8] G. Audoit et al.; Plasma-FIB sample preparation for X-ray tomography of 3D-IC interconnects; ISTFA: Conference Proceedings from the 42nd International Symposium for Testing and Failure Analysis (2016), 583. 